\title{
Differential effects of BCG vaccine on immune responses induced by vi polysaccharide typhoid fever vaccination: an explorative randomized trial
}

\author{
Bastiaan A. Blok ${ }^{1,2,3} \cdot$ Rob J.W. Arts $^{1} \cdot$ Reinout van Crevel $^{1} \cdot$ Peter Aaby $^{2,3} \cdot$ Leo A.B. Joosten ${ }^{1} \cdot$ Christine S. Benn $^{2,3}$. \\ Mihai G. Netea ${ }^{1}$
}

Received: 20 September 2019 / Accepted: 12 January 2020 / Published online: 17 February 2020

(C) The Author(s) 2020

\begin{abstract}
The Vi polysaccharide typhoid fever vaccine (TFV) provides incomplete protection against typhoid fever. BCG, the vaccine against tuberculosis, can potentiate immune responses to other vaccines through induction of trained innate immunity and heterologous adaptive immunity. We performed an explorative, randomized, noncontrolled open trial to investigate whether BCG vaccination increases humoral and cellular response to TFV and whether BCG and TFV modulate nonspecific immune responses. Thirty volunteers were randomized to receive either TFV alone or BCG followed by TFV after 2 weeks. Ex vivo leukocyte responses and anti-Vi IgG antibody titers were measured 2 weeks and 3 months after TFV. BCG administration prior to TFV vaccination did not increase specific humoral or cellular immune responses to Salmonella typhi. TFV vaccination decreased pro-inflammatory responses to non-related stimuli. This effect was counteracted by prior BCG administration, which also led to decreased IL-10 and increased IL-22 responses to non-related stimuli. In an in vitro model of trained immunity TFV led to immunotolerance, which was partially reversed by BCG-induced trained immunity. BCG does not modulate adaptive immune responses to TFV but partially prevents inhibition of innate immune responses induced by TFV. Nonspecific effects of vaccines to unrelated microbial stimuli must be considered in the evaluation of their biological effects (ClinicalTrials.gov NCT02175420).
\end{abstract}

Keywords BCG $\cdot$ Typhoid fever vaccine $\cdot$ Heterologous effects

\begin{tabular}{|c|c|}
\hline \multicolumn{2}{|c|}{ Abbreviations } \\
\hline $\mathrm{BCG}$ & Bacillus Calmette-Guérin \\
\hline LPS & Lipopolysaccharide \\
\hline MVA & Modified Vaccinia Ankara \\
\hline Pam3Cys & Pam3Cys-Ser-Lys(4) \\
\hline PBMC & Peripheral blood mononuclear cell \\
\hline
\end{tabular}

Electronic supplementary material The online version of this article (https://doi.org/10.1007/s10096-020-03813-y) contains supplementary material, which is available to authorized users.

Mihai G. Netea

mihai.netea@radboudumc.nl

$1 \quad$ Department of Internal Medicine and Radboud Center for Infectious Diseases (RCI), Radboud University Medical Center, 6526, GA Nijmegen, The Netherlands

2 Research Center for Vitamins and Vaccines, Bandim Health Project, Statens Serum Institut, DK-2300 Copenhagen, Denmark

3 Odense Patient Data Explorative Network, University of Southern Denmark/Odense University Hospital, DK-5000 Odense, Denmark
PBS Phosphate-buffered saline

TFV Typhoid fever vaccine

\section{Introduction}

Typhoid fever is a significant health problem in low-income countries and in returning travelers in high-income countries [1-3]. There are two available vaccines for protection against infection with Salmonella typhi (S. typhi): the live attenuated oral Ty21a vaccine, containing the attenuated Ty21a strain of S. typhi, and the non-live parenteral Vi capsular polysaccharide vaccine, a subunit vaccine made from purified Vi capsular polysaccharide (typhoid fever vaccine; TFV). Several trials have shown suboptimal efficacy of these vaccines, with the Ty21a and Vi polysaccharide vaccines conferring 3-year cumulative protective efficacies of $48 \%$ and $55 \%$, respectively [4]. Whereas the oral Ty $21 \mathrm{a}$ vaccine confers protection by inducing both antibody formation and cell mediated immune responses, protection against infection with $S$. typhi after Vi 
polysaccharide vaccination is thought to be generated mainly by formation of anti-Vi specific IgG antibodies [5, 6].

Bacille Calmette-Guérin (BCG) vaccine is used for the protection against tuberculosis. Apart from its protective effect against tuberculosis, BCG has also been shown to confer protection against mortality and morbidity due to all-cause mortality in low-birth weight children [7]. The mechanism behind this nonspecific effect of BCG vaccination is hypothesized to involve the induction of memory properties of the innate immune system, also known as trained immunity and induction of heterologous lymphocyte responses [8, 9]. BCG vaccination of adults as well as children leads to increased ex vivo cytokine responses to non-mycobacterial antigens several weeks after vaccination $[10,11]$. BCG has also been shown to increase adaptive antibody response to concurrent or subsequent vaccinations, such as hepatitis B vaccine, pneumococcal vaccine, and influenza vaccine [12-14].

Considering these beneficial nonspecific effects of BCG vaccination, we hypothesized that $B C G$ may potentiate the induction of innate and/or adaptive immune responses induced by the Vi capsular polysaccharide Salmonella vaccine. The present study has three aims: first, to investigate whether BCG vaccination increases the adaptive immune response to Vi polysaccharide vaccine; second, to investigate whether TFV modulates the innate immune response to heterologous (i.e., non-Salmonella) microbial ligands (innate immune

\section{a}

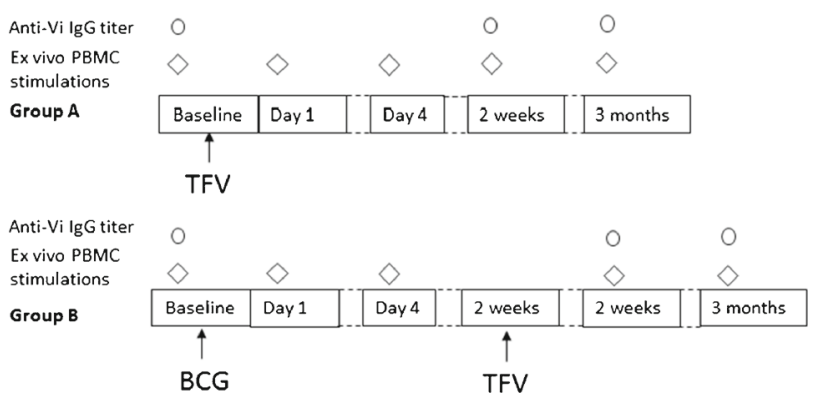

C

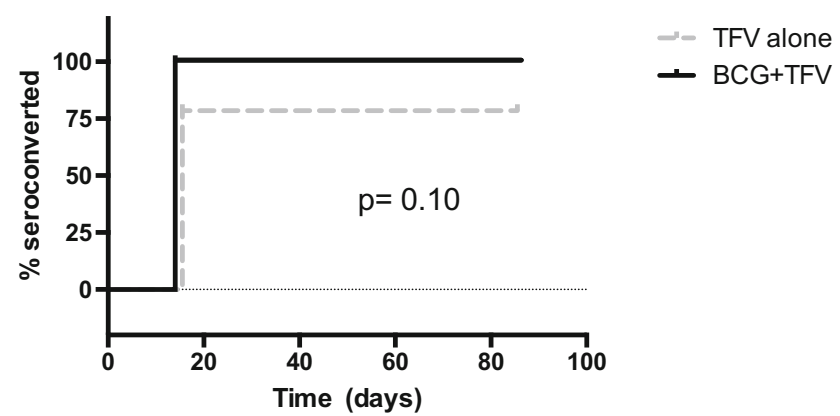

Fig. 1 a Overview of study procedures. b Absolute anti-Vi IgG antibody titer at 2 weeks and 3 months after TFV in subjects vaccinated with either TFV alone or BCG prior to TFV. Mann-Whitney U-test of antibody levels at the same time point between groups. $\mathbf{c}$ Rate of seroconversion in memory); and third, to assess whether BCG can modulate these potential nonspecific effects induced by TFV vaccine.

\section{Methods}

\section{Study design}

This study was an explorative, single-center, randomized, noncontrolled open trial performed from September 2013 to July 2015. Healthy adult volunteers with no history of prior BCG or TFV vaccination, and no known pathology at the time of inclusion, were eligible to participate. Subjects using medication and those who lived in $S$. typhi endemic areas were not eligible for participation. Informed consent was signed, and subjects were randomized in order of enrollment by alternating assignment to receive TFV alone (group A), or to receive BCG followed 2 weeks later by TFV (group B). Blood was drawn before, 1 day ( $24 \mathrm{~h} \pm 5 \mathrm{~h}$ ) and 4 days after the first vaccination (BCG for group A; TFV for group B) and subsequently 2 weeks and 3 months after TFV (Fig. 1a). The protocol was approved by the Arnhem-Nijmegen Ethical Committee, and the study was conducted in accordance of the declaration of Helsinki. Subjects who were eligible to receive $\mathrm{BCG}$ and TFV due to work or travel in endemic countries as well as normal healthy volunteers were included.

b

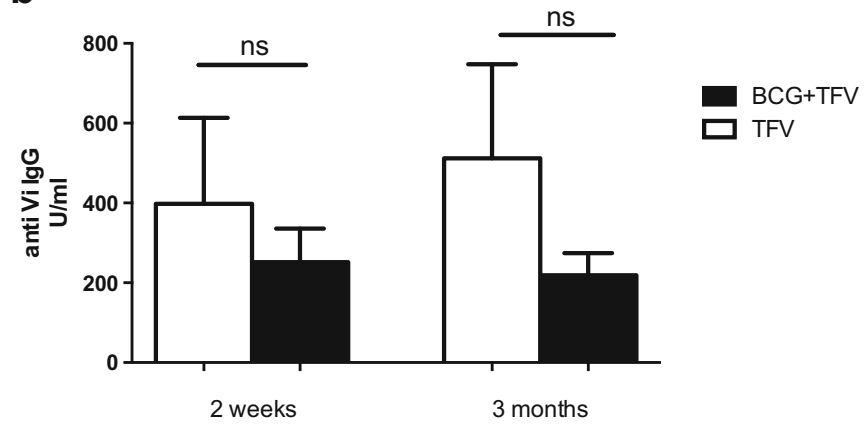

d

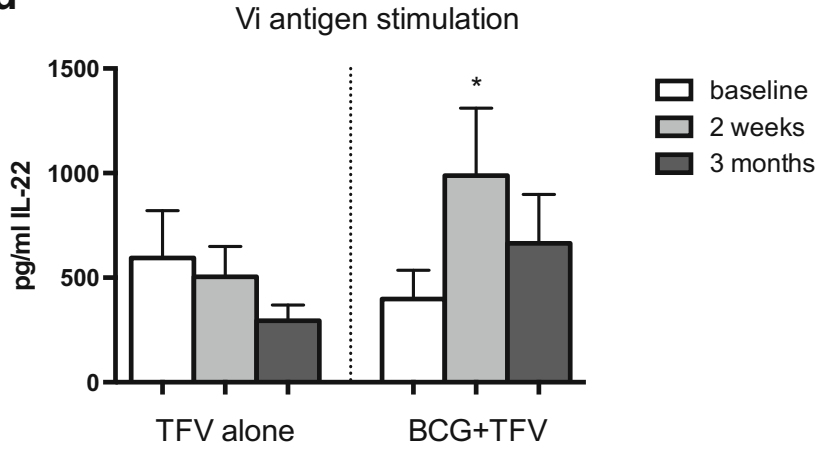

subjects vaccinated with either TFV alone or BCG prior to TFV. Fisher's exact test. $N=29$; $p<0.05$. d Ex vivo IL-22 production of PBMCs stimulated with Vi antigen. Wilcoxon signed-rank test 
At first, 20 subjects were included between February 2014 and May 2014 and followed for the study period after which data was analyzed. Since we set up this trial as an explorative study, a borderline significant result on seroconversion prompted us to include ten additional subjects from February 2015 to April 2015, of whom one was excluded after day 1 . The exact same experiments were performed in the additional volunteers. Data for 29 available subjects are presented. The trial was registered at clinicaltrials.gov (NCT02175420).

Participants were vaccinated by experienced nurses at the Radboud Travel Clinic (Radboudumc, Nijmegen, The Netherlands). All participants were vaccinated with Typhim Vi (Sanofi Pasteur, SA) using a standard dose of $0.5 \mathrm{ml}$ intramuscularly containing $0.05 \mathrm{mg} / \mathrm{ml}$ purified Vi capsular polysaccharide of $S$. typhi Ty2 strain. Participants randomized to receive BCG vaccine were vaccinated with BCG Danish 1331 (Staten Serum Institut, Denmark) using a standard dose of $0.1 \mathrm{ml}$ intradermally containing between 2 million and 8 million viable CFU per $\mathrm{ml}$.

\section{PBMC isolation and stimulation}

PBMCs were isolated using density-gradient separation over Ficoll-Paque (GE Healthcare, UK). Briefly, whole blood was diluted 1:1 with PBS, and PBMCs were separated using Ficoll. Cells were washed three times with cold PBS and resuspended in RPMI-1640 (Invitrogen) supplemented with gentamycin (50 $\mathrm{gg} / \mathrm{ml})$, glutamax $(2 \mathrm{mM})$, and pyruvate $(1 \mathrm{mM})$. Cells were counted using a Coulter counter and adjusted to $5 \times 10^{6} \%$ $\mathrm{ml}$. A total number of $5 \times 10^{5} \mathrm{PBMCs}$ in an end volume of $200 \mu \mathrm{l}$ were added to 96 -well round-bottom plates (Corning). Cells were incubated at $37{ }^{\circ} \mathrm{C}$ and $5 \% \mathrm{CO} 2$ for $24 \mathrm{~h}(\mathrm{TNF}-\alpha$, IL-1 $\beta$, IL-6), 48 h (IFN- $\gamma$, IL-10), or 7 days (IL-17, IL-22) after which supernatants were collected and stored at $-20{ }^{\circ} \mathrm{C}$ until analysis. Ten percent human pool serum was added for the 7day stimulation assays. PBMCs were cultured with RPMI (negative control), LPS (10 ng/ml, Sigma-Aldrich), heat-killed Staphylococcus aureus $\left(1 \times 10^{6} / \mathrm{ml}\right.$; S. aureus $)$, heat-killed Candida albicans conidia strain UC $820\left(1 \times 10^{6} / \mathrm{ml}\right.$; C. albicans), sonicated Mycobacterium tuberculosis $\mathrm{H} 37 \mathrm{Rv}$ $(10 \mu \mathrm{g} / \mathrm{ml} ;$ M. tuberculosis), heat-killed Escherichia coli $(1 \times$ $10^{6} / \mathrm{ml} ;$ E. coli $)$, or Typhim Vi antigen $(0,005 \mathrm{mg} / \mathrm{ml}$, Sanofi Pasteur). Human pool serum was obtained from the hematology department of Radboudumc, Nijmegen.

\section{Assessment of serological response to typhoid fever vaccination}

Before, 2 weeks and 3 months after typhoid fever vaccination, blood was collected for serology. Serum was stored at $-20{ }^{\circ} \mathrm{C}$ until analysis. Anti-Vi IgG antibody levels were measured using ELISA (VaccZyme Salmonella Typhi Vi IgG kit, The
Binding Site, Birmingham, UK) according to instructions of the manufacturer. Antibody titers are expressed as $\mathrm{U} / \mathrm{ml}$.

\section{ELISA measurements of cytokines}

Cytokines were measured in supernatants of stimulated PBMCs using commercially available ELISA kits from R\&D Systems, USA (IL- $1 \beta$, TNF- $\alpha$, IL-22, IL-17), or Sanquin, The Netherlands (IL-6, IL-10, IFN- $\gamma$ ) according to the manufacturers' instructions. All samples from one subject were measured on the same ELISA plate to avoid artifacts from batch variation between ELISA plates.

\section{In vitro trained immunity model}

To investigate the effects of BCG and TFV in vitro, a trained immunity model was used as previously described [10, 15]. Buffy coats from healthy donors were obtained after written informed consent (Sanquin blood bank, Nijmegen, The Netherlands). PBMCs were isolated using densitygradient separation over Ficoll-Paque as described above. Monocytes were obtained by density-gradient separation over Percoll, followed by 1 hour of adherence to 96-well flat bottom culture plates (Corning), after which cells were washed once and cultured for $24 \mathrm{~h}$ with either BCG $10 \mu \mathrm{g} / \mathrm{ml}$ (SSI, Denmark), Typhim Vi (0,5 $\mu \mathrm{g} / \mathrm{ml}$; Sanofi Pasteur), a combination of both or culture medium (RPMI 1640 supplemented with gentamycin, GlutaMAX and pyruvate) in an end volume of $200 \mu \mathrm{l}$. After $24 \mathrm{~h}$ of stimulation at $37^{\circ} \mathrm{C}$ and $5 \% \mathrm{CO} 2$, cells were washed once with warm PBS, and RPMI supplemented with $10 \%$ human pool serum was added to an end volume of $200 \mu \mathrm{l}$. At day 6, cells were cultured with culture medium, LPS (10 ng/ml; Sigma-Aldrich, St. Louis, MO, USA) or Pam3Cys (10 $\mathrm{gg} / \mathrm{ml}$ EMC Microcollections, Tuebingen, Germany) for $24 \mathrm{~h}$ at $37^{\circ} \mathrm{C}$ and $5 \% \mathrm{CO} 2$ after which plates were centrifuged and supernatants were stored at $-20{ }^{\circ} \mathrm{C}$ until cytokines were measured. LPS and Pam3Cys were used since they have been tested in previous studies using this model.

\section{Statistical analyses}

Differences in cytokine production and antibody titers between study groups were compared with a T-test or MannWhitney U-test depending on the distribution of the data. Differences in the proportion of subjects that reached seroconversion in the different study groups were compared using Fisher's exact test. To assess the effect of BCG and TFV on ex vivo cytokine production, data before and after vaccination were compared using a paired T-test or Wilcoxon signed-rank test depending on distribution of the data. Results are described as cytokine production to a certain stimulus before vaccination compared to cytokine production to the same stimulus at different time points after vaccination. Data was 
analyzed using GraphPad Prism, version 6.0 (GraphPad Software, San Diego, California). A $p$ value of $<0.05$ was considered statistically significant. Since this was an explorative study, no correction for multiple testing was performed. All data are expressed as mean \pm SEM unless stated otherwise.

\section{Results}

\section{Characteristics of study population and side effects}

A total of 30 volunteers were randomized for vaccination, 15 to TFV (group A) and 15 to BCG followed by TFV (group B). One subject from study group A was excluded after day 1 because of a vaccination history that included previous TFV. The majority of subjects were female (group A: 12/14; group B: 13/15); median age was similar in both groups (21 years, range 20-27). All participants in the BCG group reported erythema, minor swelling, and tenderness at injection site, which resolved within 1 month. After TFV vaccination, only transient pain at the injection site was reported.

\section{Prior BCG vaccination does not increase adaptive response to TFV}

\section{Humoral antibody response}

To investigate the potentiating effect of $\mathrm{BCG}$ vaccination on the antibody response to TFV, we measured absolute $\operatorname{IgG}$ antibody titers to Salmonella Vi antigen at baseline, 2 weeks and 3 months after TFV, as well as the proportion of subjects with seroconversion (defined as a fourfold increase in antibody titer compared to baseline) at 2 weeks and 3 months after TFV in both groups. Baseline levels of anti-Vi IgG were either very low or undetectable in both groups, with no difference between the groups. The absolute anti-Vi IgG titer 2 weeks and 3 months after TFV was higher in subjects who did not receive prior BCG, although this effect was not statistically significant and mainly caused by one very high responder in the TFV alone group (Fig. 1b).

There was no significant difference in seroconversion at 2 weeks in subjects that received BCG prior to TFV compared to those who only received TFV; this was seen in $15 / 15$ in the BCG + TFV groups versus 11/14 in the TFV alone group (Fig. 1c). This effect was sustained at the 3-month follow-up time point (Fisher's exact test, $p=0.10$ ).

\section{Typhoid fever specific cytokine responses}

We assessed whether TFV or BCG induced short-term T cell responses and trained immunity by assessing cytokine production capacity to either TFV or unrelated stimuli. Cytokine responses after PBMC in vitro stimulation with TFV were poor, with no induction of innate cytokines (TNF- $\alpha$, IL-6, IL-1 $\beta$, IL-10) or adaptive cytokines IFN- $\gamma$ or IL-17 (data not shown). BCG prior to TFV led to a significant increase in IL-22 production 2 weeks after TFV, but this effect was not sustained at 3 months (Fig. 1d).

\section{Short-term modulation of adaptive and innate immune responses by TFV and BCG vaccination}

TFV vaccination increased pro-inflammatory cytokine production (IL-1 $\beta$, IFN- $\gamma$, and TNF- $\alpha$, IL-6) upon stimulation with LPS, S. aureus, $C$. albicans, and M. tuberculosis 1 day after vaccination (Fig. 2a, b and supplementary Fig. 1A, B) compared to before vaccination. This increase in cytokine production returned to baseline level 4 days after vaccination. In contrast, no effects were observed on IL-10, IL-17, or IL-22 production (supplementary Fig. 1C-E). After BCG vaccination production of IFN- $\gamma$, IL- $1 \beta$, and IL- 6 to heterologous stimuli at day 1 was increased compared to before vaccination, which returned to baseline at day 4 (Fig. 2a, b and supplementary Fig. 1A, B), although the effects did not reach statistical significance in about half of the cytokine-antigen combinations. No effects were observed on TNF- $\alpha$, IL-10, IL-17, or IL-22 production (supplementary Fig. 1C-E).

\section{TFV induces long-term innate immune tolerance, which is partially reversed by BCG}

We assessed long-term induction of trained immunity by comparing cytokine responses to unrelated microbial ligands at baseline to responses 2 weeks and 3 months after TFV vaccination. TFV vaccination was associated with significantly decreased TNF- $\alpha$, IL- $1 \beta$, and IL- 6 responses upon stimulation with LPS, as well as significantly decreased IL6 production to stimulation with $C$. albicans: a de facto induction of immune tolerance (Fig. 3a and supplementary Fig. 2A, B), although it was striking that TNF- $\alpha$ production to $C$. albicans was upregulated 2 weeks after vaccination. TNF- $\alpha$ and IL- $1 \beta$ production upon stimulation with $S$. aureus and $M$. tuberculosis were also decreased 3 months after vaccination, but this effect was not statistically significant (supplementary Fig. 2a, b). IL-6 production to $S$. aureus was not affected (data not shown). The production of the anti-inflammatory cytokine IL-10 was decreased at 3 months after TFV, and for stimulation with $C$. albicans and M. tuberculosis, this reached statistical significance (Fig. 3b). No effects were observed on IFN- $\gamma$ and IL-17 responses, but IL-22 production upon stimulation with S. aureus was significantly decreased 3 months after TFV vaccination (Fig. 3c, d, supplementary Fig. 2C).

Individuals who received $\mathrm{BCG}$ prior to TFV vaccination showed partial abrogation of reduction in production of TNF- $\alpha$, IL- $1 \beta$, and IL- 6 following TFV vaccination 
Fig. 2 Ex vivo production of IL$1 \beta$ (a) and IFN- $\gamma(\mathbf{b})$ by PBMCs stimulated with LPS, heat-killed S. aureus (S.a), heat-killed

C. albicans $(C . a)$, and sonicated M. tuberculosis (M.tb) at baseline, and at 1 and 4 days after vaccination with TFV or BCG vaccine.

Wilcoxon signed-rank test comparing values before and after vaccination with the same stimulus; $\mathrm{N}=29, \wedge p=0.06, * p<0.05$ $* * p<0.01$

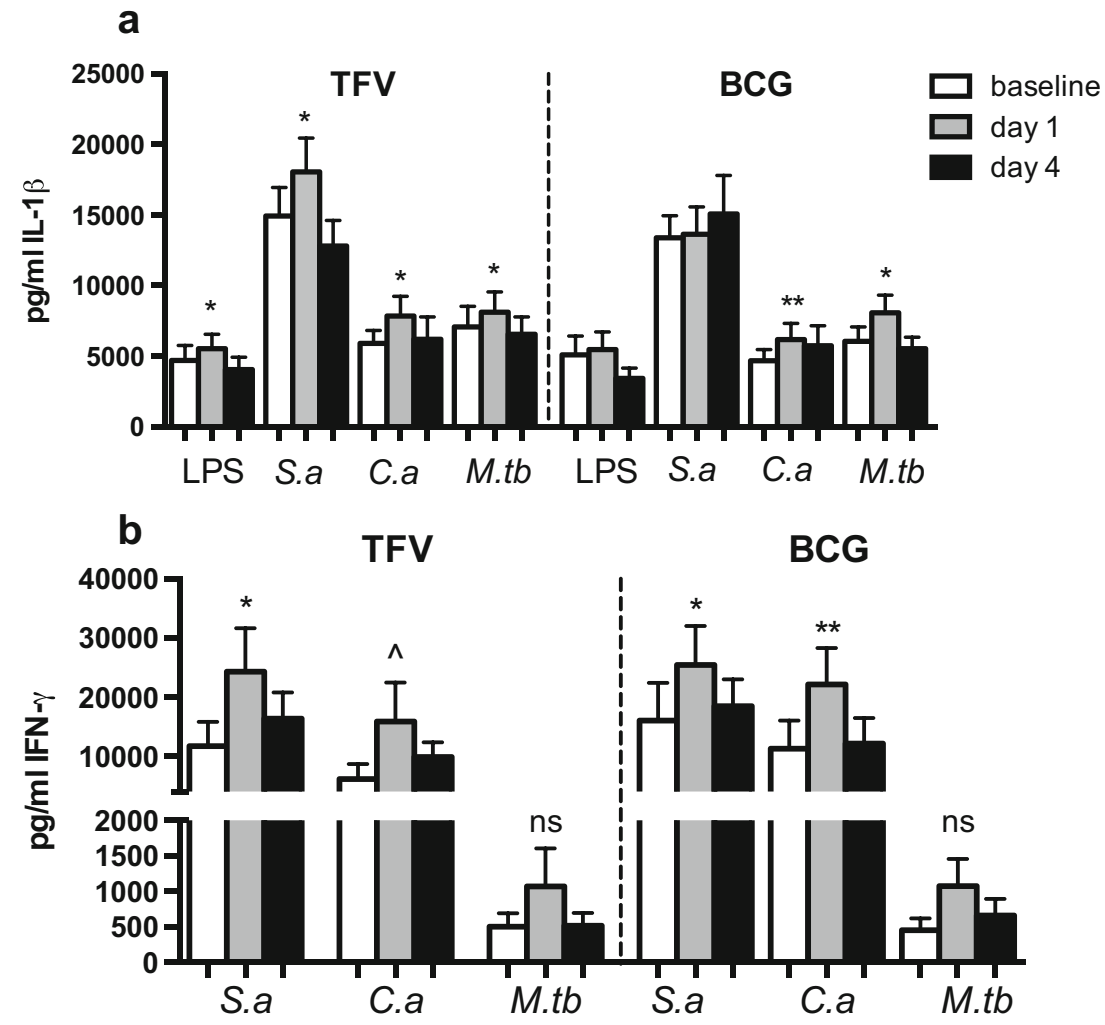

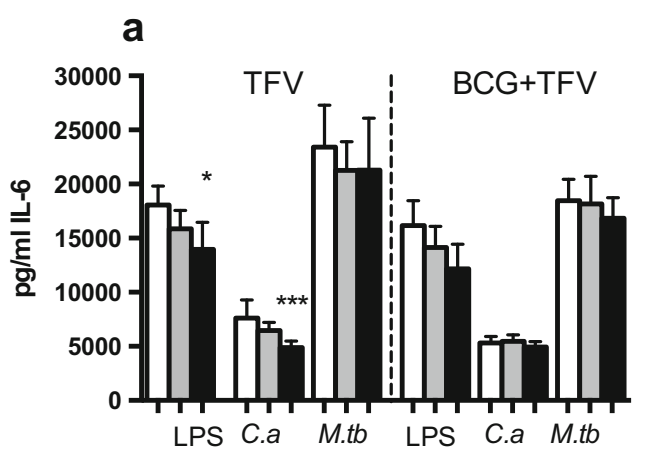

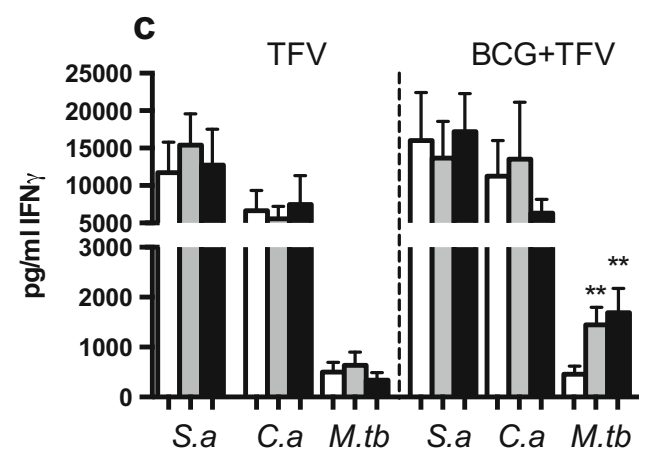

Fig. 3 Ex vivo production of IL-6 (a), IL-10 (b), IFN- $\gamma(\mathbf{c})$, and IL-22 (d) by PBMCs stimulated with LPS, heat-killed $S$. aureus (S.a), heat-killed C. albicans (C.a), and sonicated M. tuberculosis (M.tb), before TFV or BCG vaccination (baseline) and at 2 weeks and 3 months after TFV
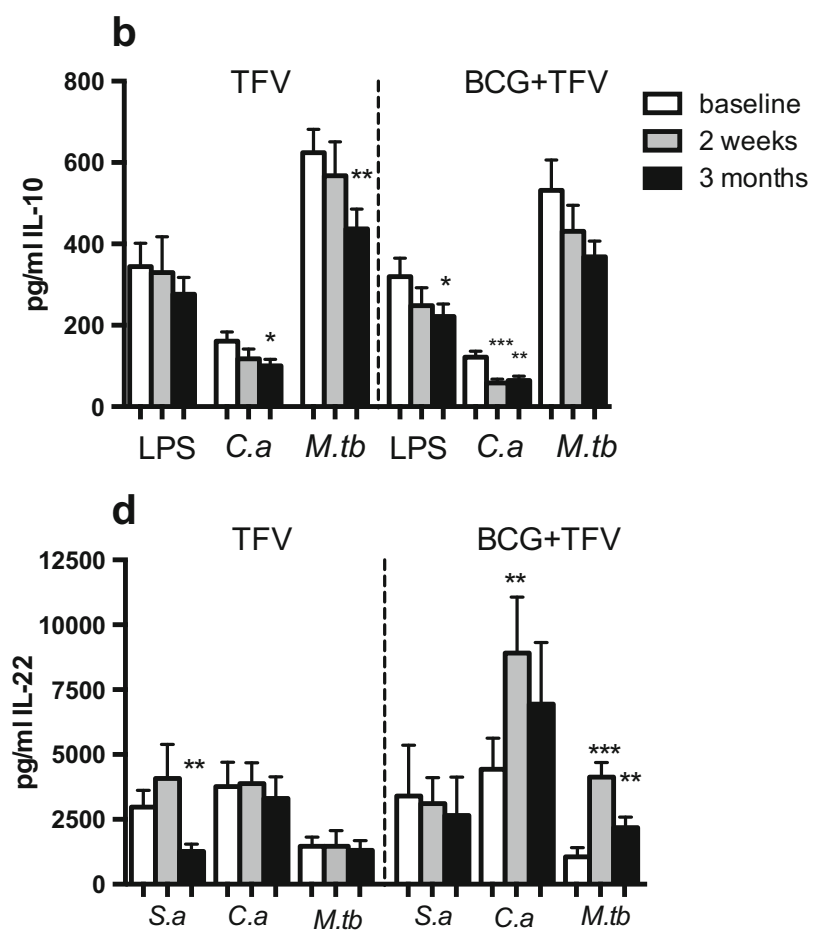

vaccination. Wilcoxon signed-rank test comparing values before and after vaccination with the same stimulus; $\mathrm{N}=29, * p<0.05$, ** $p<0.01$, *** $p<0.001$ 
(Fig. 3a, supplementary Fig. 2A, B). BCG also counteracted inhibition of IL-6 production to $C$. albicans stimulation following TFV vaccination. Interestingly, BCG administered prior to TFV led to significant decreases in IL-10 production to LPS, S. aureus, and C. albicans at 2 weeks and 3 months after TFV vaccination (Fig. 3b). As expected, subjects who received BCG prior to TFV showed significantly increased IFN- $\gamma$ and IL-22 production to M. tuberculosis 2 weeks and 3 months after TFV (Fig. 3c, d). Strikingly, BCG prior to TFV led to significantly increased IL-22 responses to stimulation with $C$. albicans and $E$. coli and abrogated the decrease in IL22 production to $S$. aureus observed in the TFV alone group (Fig. 3d and supplementary Fig. 2D).

\section{TFV induces immune tolerance in an in vitro model}

To further validate the immunomodulatory effects of TFV, we used a well-defined in vitro model of trained immunity (Fig. 4a). When monocytes were primed for $24 \mathrm{~h}$ with TFV and rechallenged with TLR4 ligand LPS or TLR2 ligand Pam3Cys 1 week afterwards, IL-6 production was significantly decreased. Interestingly, when BCG was added to TFV during the first $24 \mathrm{~h}$ of priming, this effect was partially reversed (Fig. 4b), in line with the effects observed in the clinical trial.

\section{Discussion}

We investigated whether BCG vaccination increases the humoral and cellular response to TFV and whether BCG and TFV modulate the immune response to non-related antigens.
BCG vaccination prior to TFV did not affect absolute $S$. typhi antibody titers, and although seroconversion was more common in the BCG group, this failed to reach statistical significance. We did observe a transiently increased cellular recall IL-22 response to TFV, although it should be noted that due to the 2-week interval between the two vaccines and the known kinetics of immune responses elicited by BCG, this might have been an effect purely elicited by BCG without it affecting the response to TFV. A similar trend to improved seroconversion to influenza vaccine after previous BCG vaccination was observed in an earlier study from our group [14]. BCG has been shown to affect innate and $\mathrm{T}$ cell-mediated immune responses to protein, peptide, or conjugated polysaccharide vaccines, which behave as T-dependent antigens [12-14]. In contrast, BCG might not affect the response to $\mathrm{TFV}$, a capsular polysaccharide vaccine, which acts as a $\mathrm{T}$ cell-independent antigen [16]. A second possibility is that the batch of BCG used in this study was less immunogenic compared to batches used in previous studies, as batch-tobatch variation in immunogenicity and capacity for inducing trained immunity has been observed previously [17].

In addition to specific induction of lymphocyte responses, vaccines also modulate nonspecific innate immune responses $[10,11]$. In this study BCG and TFV induced transient, shortterm increased pro-inflammatory cytokine production to unrelated stimuli. This effect probably reflects an acute-phase reaction, since trained immunity needs days to weeks to be established [18].

On a longer term, 2 weeks and 3 months after TFV vaccination, innate pro-inflammatory cytokine production to LPS was decreased: a de facto innate immune tolerance effect. This effect was abrogated when BCG was given prior to TFV.
Fig. 4 a In vitro model of trained immunity. b Monocytes were primed with RPMI, BCG, Typhim Vi, or BCG + Typhim Vi for $24 \mathrm{~h}$. Stimuli were washed off, and cells were kept in culture for 6 days, after which they were restimulated with LPS or Pam3Cys for 24 H. $I L-6$ was measured in supernatants. Wilcoxon signed-rank test, $N=8$, $* p<0.05, * * p<0.01$
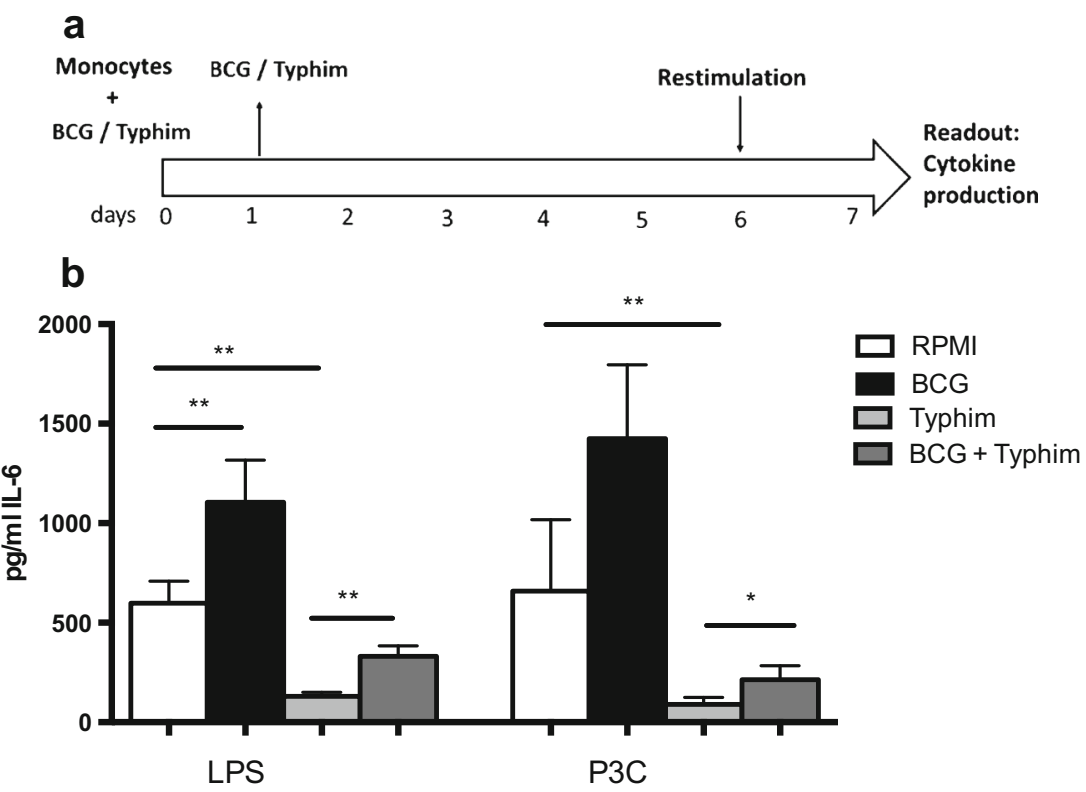
Furthermore, in these subjects, we observed a decrease of the anti-inflammatory cytokine IL-10 to stimulation with LPS, suggesting that BCG skews the balance towards a more proinflammatory response to LPS, although TFV may also have contributed to this effect. It is likely that this opposing effect of BCG is due to its capacity to induce trained immunity and thus counteract immune tolerance [19]. Another possibility is that viable BCG was present at the time of TFV vaccination and that the ongoing immune response modulated the immunetolerizing effect of TFV. Since inflammatory monocytes are important in the initial immune response to $S$. typhi [20], it could be speculated that BCG vaccination would lead to a more efficient immune response to typhoid fever. Future studies are warranted to investigate the effects of BCG vaccination on the incidence or outcome of typhoid fever.

This may be the first study describing heterologous effects of the Vi polysaccharide typhoid vaccine in a human in vivo setting, but there are earlier data to support two possible explanations for this immunosuppressive effect of TFV. First, earlier studies found that Vi polysaccharide inhibits monocyte and $\mathrm{T}$ cell activation, leading to reduced heterologous cytokine responses $[18,21]$. Since we observed immunosuppressive effects of TFV in an in vitro model of trained immunity, it most likely has a direct effect on monocyte function. Second, the immunomodulatory effect of TFV may be mediated by sialylated IgG antibodies, which are induced upon immunization with T cell-independent antigens and have been shown to have immunosuppressive actions [22].

An immunosuppressive effect of the non-live TFV is in line with previous immunological studies that have shown immunosuppressive effects of the inactive influenza vaccine [14], MVA [23], and diphtheria-tetanus-pertussis vaccine [24]. These effects are in contrast to the immune-stimulatory effects observed after the live BCG [10] and smallpox vaccines [23]. In parallel, an increasing number of epidemiological studies from low-income settings show that non-live vaccines, while protective against the target disease, may increase mortality from non-related causes $[25,26]$, while live vaccines reduce overall mortality more than can be explained by target disease prevention [7, 27]. While simplistic, it is tempting to speculate that the immunosuppressive and immune-stimulatory effects observed after vaccination with non-live and live vaccines, respectively, explain why the vaccines seem to have so different effects on overall mortality in real life.

We did not find increased pro-inflammatory cytokine responses to unrelated antigens, also known as trained immunity, in subjects that received BCG prior to TFV, in contrast to previous studies $[10,11,28]$. This might be explained by batch variation in the BCG vaccine [21], or due the fact that the immune-tolerizing effect of TFV opposed the innate training effects of BCG. Although we did not find increased proinflammatory cytokine responses, we observed reduced production of IL-10, suggesting that the overall balance of the immune response is more pro-inflammatory. This is in line with a previous study which found decreased IL-10 responses after BCG vaccination in low-birth weight infants [11]. Furthermore, we found heterologous effects of BCG on T cell immunity in the form of increased IL-22 responses to $E$. coli and $C$. albicans, in line with previous studies of BCG showing strong effects on heterologous IL-22 responses [28, 29].

Several limitations to our study should be noted. With initially ten subjects per study group of TFV alone or BCG + TFV, the sample size is small. Ten additional volunteers (five individuals/groups) were recruited after observing a potential effect on seroconversion. However, the final conclusion after analyzing the data in the entire group of vaccinated individuals was that BCG did not impact the serological effects of typhoid fever vaccine. Another limitation of the study is that the reported $p$ values were not corrected for multiple testing, due to the explorative nature of this study.

In conclusion, BCG vaccination does not enhance immunogenicity of TFV vaccination, but larger studies are needed to examine a possible moderate increase in antibody responses. The Vi polysaccharide typhoid fever vaccine has nonspecific immuno-tolerizing effects in vitro and in vivo, which are partially abrogated by prior BCG vaccination. This finding supports the concept that both live and non-live vaccines have important nonspecific immunomodulatory effects, which influence the immune response to other microorganisms and may influence overall morbidity and mortality.

Acknowledgments We thank Charlotte van Daal, Jeanine Leijser, and Inge Verhagen-Stolk, from the Radboud travel clinic, for help with recruitment of volunteers and performing vaccinations in this study.

Funding information This work was supported by the European Research Council (ERC \#310372 to MGN), the Netherlands Organization for Scientific Research (NWO Spinoza Grant to MGN), and the Danish National Research Foundation (DNRF108 to CVIVA).

\section{Compliance with ethical standards}

Potential conflicts of interest The salaries of BAB, PA, and CSB are administered, but not financed by SSI, the producer of the BCG vaccine used in the present study. RJW, RvC, LJ, and MGN declare no conflict of interest. All authors have approved the final article.

Open Access This article is licensed under a Creative Commons Attribution 4.0 International License, which permits use, sharing, adaptation, distribution and reproduction in any medium or format, as long as you give appropriate credit to the original author(s) and the source, provide a link to the Creative Commons licence, and indicate if changes were made. The images or other third party material in this article are included in the article's Creative Commons licence, unless indicated otherwise in a credit line to the material. If material is not included in the article's Creative Commons licence and your intended use is not permitted by statutory regulation or exceeds the permitted use, you will need to obtain permission directly from the copyright holder. To view a copy of this licence, visit http://creativecommons.org/licenses/by/4.0/. 


\section{References}

1. Crump JAA, Mintz EDD (2010) Global trends in typhoid and paratyphoid fever. Clin Infect Dis 50:241-246. https://doi.org/10.1086/ 649541

2. Steffen R, Behrens RH, Hill DR, Greenaway C, Leder K (2015) Vaccine-preventable travel health risks: what is the evidence-what are the gaps? J Travel Med 22:1-12. https://doi.org/10.1111/jtm. 12171

3. Dave J, Sefton A (2015) Enteric fever and its impact on returning travellers. Int Health 7:163-168. https://doi.org/10.1093/inthealth/ ihv018

4. Anwar E, Goldberg E, Fraser A, Acosta Camilo J, Paul M, Leibovici L 2014 Vaccines for preventing typhoid fever. Cochrane Database Syst Rev Volume|. https://doi.org/10.1002/ 14651858.CD001261.pub3

5. Klugman KP, Koornhof HJ, Robbins JB, Le Cam NN, Le Cams NN, Robbins JB (1996) Immunogenicity, efficacy and serological correlate of protection of Salmonella typhi vi capsular polysaccharide vaccine three years after immunization. Vaccine 14:435-438

6. World Health Organization 2011 The Immunological Basis for Immunization Series: module 20: Salmonella enterica serovar Typhi (typhoid) vaccines. World Health Organization. https:// apps.who.int/iris/handle/10665/44752

7. Aaby P, Roth A, Ravn H, Napirna BM, Rodrigues A, Lisse IM et al (2011) Randomized trial of BCG vaccination at birth to low-birthweight children: beneficial nonspecific effects in the neonatal period? J Infect Dis 204:245-252. https://doi.org/10.1093/infdis/jir240

8. Blok BA, Arts RJW, van Crevel R, Benn CS, Netea MG (2015) Trained innate immunity as underlying mechanism for the longterm, nonspecific effects of vaccines. J Leukoc Biol 98:347-356. https://doi.org/10.1189/jlb.5RI0315-096R

9. Goodridge HS, Ahmed SS, Curtis N, Kollmann TR, Levy O, Netea MG et al (2016) Harnessing the beneficial heterologous effects of vaccination. Nat Rev Immunol 16:392-400. https://doi.org/10. 1038/nri.2016.43

10. Kleinnijenhuis J, Quintin J, Preijers F, Joosten LA, Ifrim DC, Saeed $\mathrm{S}$ et al (2012) Bacille Calmette-Guerin induces NOD2-dependent nonspecific protection from reinfection via epigenetic reprogramming of monocytes. Proc Natl Acad Sci U S A 109: 17537-17542. https://doi.org/10.1073/pnas.1202870109

11. Jensen KJ, Larsen N, Sorensen SB, Andersen A, Eriksen HB, Monteiro I et al (2015) Heterologous immunological effects of early BCG vaccination in low-birth-weight infants in Guinea-Bissau: a randomized-controlled trial. J Infect Dis 211:956-967. https://doi. org/10.1093/infdis/jiu508

12. Ota MOC, Vekemans J, Susanna E, Fielding K, Sanneh M, Kidd M et al (2002) Influence of Mycobacterium bovis Bacillus CalmetteGuerin on antibody and cytokine responses to human neonatal vaccination 1. J Immunol 168:919-925

13. Ritz N, Mui M, Balloch A, Curtis N (2013) Non-specific effect of Bacille Calmette-Guerin vaccine on the immune response to routine immunisations. Vaccine 31:3098-3103. https://doi.org/10.1016/j. vaccine.2013.03.059

14. Leentjens J, Kox M, Stokman R, Gerretsen J, Diavatopoulos DA, van Crevel $R$ et al (2015) BCG vaccination enhances the immunogenicity of subsequent influenza vaccination in healthy volunteers: a randomized, placebo-controlled pilot study. J Infect Dis 212: 1930-1938. https://doi.org/10.1093/infdis/jiv332

15. Bekkering S, Blok BA, Joosten LAB, Riksen NP, van Crevel R, Netea MG (2016) In-vitro experimental model of trained innate immunity in human primary monocytes. Clin Vaccine Immunol 23:926-933. https://doi.org/10.1128/CVI.00349-16
16. Marshall JL, Flores-Langarica A, Kingsley RA, Hitchcock JR, Ross EA, López-Macías C et al (2012) The capsular polysaccharide vi from Salmonella typhi is a B1b antigen. J Immunol 189:55275532. https://doi.org/10.4049/jimmunol.1103166

17. Biering-Sørensen S, Jensen KJ, Aamand SH, Blok B, Andersen A, Monteiro I et al (2015) Variation of growth in the production of the BCG vaccine and the association with the immune response. An observational study within a randomised trial. Vaccine 33:20562065. https://doi.org/10.1016/j.vaccine.2015.02.056

18. Santhanam SK, Dutta D, Parween F, Qadri A (2014) The virulence polysaccharide vi released by Salmonella Typhi targets membrane prohibitin to inhibit T-cell activation. J Infect Dis 210:79-88. https://doi.org/10.1093/infdis/jiu064

19. Netea MG, Joosten LAB, Latz E, Mills KHG, Natoli G, Stunnenberg HG et al (2016) Trained immunity: A program of innate immune memory in health and disease. Science 352: aaf1098-aaf1098. https://doi.org/10.1126/science.aaf1098

20. Griffin AJ, McSorley SJ (2011) Development of protective immunity to Salmonella, a mucosal pathogen with a systemic agenda. Mucosal Immunol 4:371-382. https://doi.org/10.1038/mi.2011.2

21. Garg R, Akhade AS, Yadav J, Qadri A (2015) MyD88-dependent pro-inflammatory activity in vi polysaccharide vaccine against typhoid promotes Ab switching to IgG. Innate Immun 21:778-783. https://doi.org/10.1177/1753425915599242

22. Hess C, Winkler A, Lorenz AK, Holecska V, Blanchard V, Eiglmeier $\mathrm{S}$ et al (2013) T cell-independent B cell activation induces immunosuppressive sialylated IgG antibodies. J Clin Investig Clin 123:3788-3796. https://doi.org/10.1172/JCI65938. tosylated

23. Blok BA, Jensen KJ, Aaby P, Fomsgaard A, van Crevel R, Benn CS et al (2019) Opposite effects of Vaccinia and modified Vaccinia Ankara on trained immunity. Eur J Clin Microbiol Infect Dis 38: 449-456. https://doi.org/10.1007/s10096-018-03449-z

24. Noho-Konteh F, Adetifa JU, Cox M, Hossin S, Reynolds J, Le MT et al (2016) Sex-differential non-vaccine specific immunological effects of diphtheria-tetanus-pertussis and measles vaccination. Clin Infect Dis 63:1213-1226. https://doi.org/10.1093/cid/ciw492

25. Aaby P, Benn C, Nielsen J, Lisse IM (2012) Rodrigues a., Ravn H. testing the hypothesis that diphtheria-tetanus-pertussis vaccine has negative non-specific and sex-differential effects on child survival in high-mortality countries. BMJ Open 2:e000707-e000707. https://doi.org/10.1136/bmjopen-2011-000707

26. Aaby P, Ravn H, Roth A, Rodrigues A, Lisse IM, Diness BR et al (2012) Early diphtheria-tetanus-pertussis vaccination associated with higher female mortality and no difference in male mortality in a cohort of low birth weight children: an observational study within a randomised trial. Arch Dis Child 97:685-691. https://doi. org/10.1136/archdischild-2011-300646

27. Aaby P, Martins CL, Garly M-L, Bale C, Andersen A, Rodrigues A et al (2010) Non-specific effects of standard measles vaccine at 4.5 and 9 months of age on childhood mortality: randomised controlled trial. Bmj 341:c6495-c6495. https://doi.org/10.1136/bmj.c6495

28. Kleinnijenhuis J, Quintin J, Preijers F, Benn CS, Joosten LAB, Jacobs $C$ et al (2014) Long-lasting effects of bcg vaccination on both heterologous th1/th17 responses and innate trained immunity. J Innate Immun 6:152-158. https://doi.org/10.1159/000355628

29. Arts RJW, Blok BA, Aaby P, Joosten LAB, de Jong D, van der Meer JWM et al (2015) Long-term in vitro and in vivo effects of $y$ irradiated BCG on innate and adaptive immunity. J Leukoc Biol 98: 995-1001. https://doi.org/10.1189/jlb.4MA0215-059R

Publisher's note Springer Nature remains neutral with regard to jurisdictional claims in published maps and institutional affiliations. 